Textes und der Anmerkungen. Besonders der Aufsatz über die Grundlagen der Arithmetik (Schubert) hat in J. Tannery und J. Molk berufene Bearbeiter gefunden. Es warden hier stellenweise ganze Kapitel eingeschaltet. Die Durchsicht des zwischen den Sternen liegenden Stoffes zeigt, wie interessante und wichtige Daten besonders historischen und philosophischen Inhalts gegen die deutsche Ausgabe hinzugekommen sind. Aber auch die Aufsätze von E. Netto und von A. Pringsheim sind infolge der schönen Ergänzungen keine reine Übersetzung des dentschen Textes.

J. $P$.

Sammlung von Formeln und Sätzen aus dem Gebiete der elliptischen Funktionen nebst Anwendungen von J. Thomae in Jena. B. G. Teubner, Leipzig 1905, IV $+44 \mathrm{~S}$. Gr. $28 \mathrm{~cm}$. M. 2.80.

Der Verfasser wollte durch seine Sammlnng einem allgemeinen Bedürfnisse nachkommen and erreichte den Zweck um so mehr, als er die praktische Seite dadurch hervorkehrte, daß er zur Berechnung der elliptischen Funktionen and Integrale gut verwendbare, der hinreichenden Genauigkeit entsprechende Näherungsformeln gab. Wir können uns leider die Bemerkung nicht versagen, daß man sich unter der Fülle oft zu dicht zusammengedrängter Formeln stellenweise kaum zurechtfindet; die Raumersparnis geschieht auch hier sehr auf Rechnung der Übersichtlichkeit.

Der zweite Teil enthält verschiedene Anwendungen aus der Mechanik, Geometrie and Potentialtheorie, worunter wohl alle die wichtigsten Anwendungen der elliptischen Funktionen in diesen Gebieten sich vorfinden.

\title{
J. P.
}

Die Wissenschaft, Sammlung naturwissenschaftlicher und mathematischer Monographien; Heft 12. Die Fortschritte der kinetischen Gastheorie von Dr. G. Jäger. Friedrich Viewegund Sohn, Braunschweig 1906.

Der vorliegende Band der Monographiensammlung "Die Wissenschaft" bildet ohne Zweifel einen der wertvollsten Beiträge in der an wertvollen Beiträgen nun schon so reichen Reihe von Monographien. Doch abgesehen davon, daß hier wirklich ein Berufener zu Worte kam, einer der selbst regen Anteil am Ausbau der Gastheorie genommen, erscheint das Werk auch in einem sehr günstigen Zeitpunkte. "Scheinen ja doch", wie der Verfasser in seiner Vorrede hervorhebt, „für die kinetische Gastheorie wieder bessere Tage zu kommen" und angeregt durch die Erfolge der Atomistik in der Lehre von der Elektrizitätsleitung in Gasen wendet sich so mancher wieder den Problemen der reinen Gastheorie zu.

Was das Werk selbst anlangt, so besteht es aus einer Einleitung, die in ziemlich ausführlicher Weise einen Grundriß der kinetischen Gastheorie gibt, und einem weiteren Teil, der die fortgeschritteneren Probleme behandelt.

Wenn nun auch der Einleitung vielleicht ein etwas allzubreiter Raum zugewiesen wurde - sie füllt nahezu die Hälfte der Seitenzahl des Heftes so wird dies doch gerade der Majorität der Leser willkommen sein; denn sie alle wollen sich ja nur rasch an der Hand eines verläßlichen Führers über die Gasgesetze, Geschwindigkeiten der Moleküle, mittlere Weglänge, spezifische Wärme, innere Reibung, Wärmeleitung, Diffusion a. s. w. orientieren. Sie alle werden von dieser Einleitung hochbefriedigt sein, denn außer den genannten 
Dingen wird hier auch noch das Maxwellsche Verteilungsgesetz und der Virialbegriff in klarer, leicht verständlicher Fassung vorgebracht.

Wer jedoch das Werk vom Gesichtspunkt der „Fortschritte" in die Hand nimmt, wird nicht umhin können zu bedauern, daß sich der Verfasser mit Rücksicht auf die Einleitung jm Kern seines Werkes einschränken mußte. Hier wird zunächst der exakte $B \in$ weis des Verteilungsgesetzes nachgetragen und das H-Theorem behandelt. Des weiteren werden die Ergänzungen gebracht, die Boltzmann bezüglich der äußeren Kräfte an dem Gesetze angebracht hat und die Gültigkeit dieses Gesetzes für beliebig kleine Kraftfelder erörtert. Das nächste Kapitel bringt dann eine weitere Ausführung des Virialtheorems und daran anschließend die Herleitung der Zustandsgleichung mäßig komprimierter Gase bezw. der van-der-Waalschen Gleichung. Kapitel V befaßt sich mit der Abhängigkeit der mittleren Weglänge und des Reibungskoefizienten von der Temperatur, wobei die einschlägigen Arbeiten von Sutherland und Reinganum Beräcksichtigung finden. Kapitel VI ist cem Weiterausbau der Wärmeleitungstheorie gewidmet; hier wird speziell das Fhänomen des Temperatursprunges sehr ausführlich erörtert und finden die Arbeiten v. Smoluchowskis ihre Würdigung. Die letzten drei Abschnitte endlich sind der Theorie der idealen Flüssigkeit gewidmet und hier legt der Verfasser, auf ureigenstem Arbeitsfelde stehend, seine Ansichten äber den Gegenstand nieder.

Wie man sieht, ist die Fülle des Gebotenen auch im zweiten Teile des Heftes keineswegs gering, doch fehlt manches, worauf man nur ungern verzichtet; so wäre wohl vielen eine Darlegung der Theorie Maxwells, die die Molekel als Kraftzentra anffaßt, gewiß willkommen gewesen, um so mehr, als ja nur auf Grund dieser Theorie das Reibungsproblem exakt zu behandeln ist. Doch der Verfasser hat sich infolge mangelnden Raumes eben nur einer einzigen Anschauungsweise anschlieBen können und als solche diejenige gewählt, die die Molekeln als elastische Kugeln auffaßt; darum dürfen wir auch nicht mit ihm rechten, vielmehr hoffen, daß die nächste Auflage mehr Ranm und damit Erfüllung unserer Wünsche bringt.

J. $N$.

Die Elemente der Differential- und Integralrechnung. Von Prof. Steckelberg. B. G. Teubner, Leipzig u. Berlin 1906. $80 \mathrm{~S}$. Das Büchlein ist für den Gebrauch beim Unterricht in den beiden obersten Gymnasialklassen bestimmt und enthält das Allereinfachste in ebensolcher Darstellung. Ohne nun an die Strenge übertriebene Anforderungen zu stellen, könnte ein Mangel, wie der Schluß auf pag. 16 und 17, daß weil $\cos \Delta x=1$ für $\Delta x=0$ auch der Quotient $\sin x \frac{1-\cos \Delta x}{\Delta x}$ für $\Delta x=0$ verschwinde, leicht vermieden werden, zumal da ja die Abhilfe nahe liegt und die Anwendung derselben Schlußweise die ganze Differentialrechnung illusorisch macht. $W$.

L'Ếther, principe universel de forces. Par A. Ma rx, mémoires résumés par C. Benoit. Gauthier-Villars, Paris 1905.

Den Ausgangspunkt der Betrachtungen des Herrn Marx bildet die Hypothese Fresnels, der zufolge jedes ponderable Atom von einer Atmosphäre kondensierten Äthers eingehüllt ist. Herr Marx transformiert diese Annahme dahin, daß das ponderable Atom im Schoße des Ethers, der sich wie ein ideales Gas verhält, ein Depressionszentrum bildet, welches mit dem umgebenden 